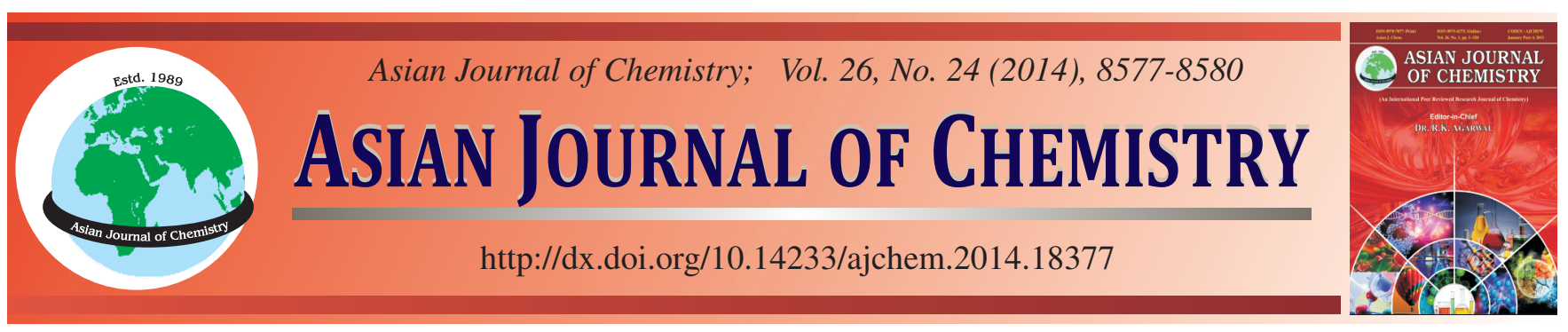

\title{
Preparation and Characterization of Reactive Type Dripping Agent Containing $\alpha$-Double Bond
}

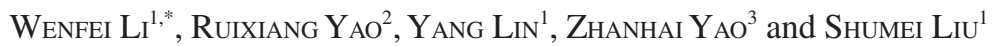

${ }^{1}$ College of Chemistry and Environmental Engineering, Changchun University of Science and Technology, Changchun 130022, P.R. China ${ }^{2}$ College of Chemistry, Jilin University, Changchun 130012, P.R. China

${ }^{3}$ State Key Laboratory of Polymer Physics and Chemistry, Changchun Institute of Applied Chemistry, Chinese Academy of Sciences, Changchun 130022, P.R. China

*Corresponding author: E-mail: liwenfei1130@126.com

Received: 26 June 2014;

Accepted: 18 September 2014;

Published online: 1 December 2014;

AJC-16407

A reactive type dripping agent containing $\alpha$ double bond, sorbitan monostearate-itaconic anhydride (Span60-IAH) was synthesized with sorbitan monostearate (Span60) and itaconic anhydride as main starting materials. The chemical structures of Span60-IAH were characterized by Fourier transform infrared (FT-IR) spectroscopy and ${ }^{1} \mathrm{H}$ NMR. The effect of reaction time on conversion of Span60-IAH was studied. The surface activity and surface tension of Span60-IAH were evaluated by a drop weight method. Thermal stability of Span60-IAH and Span60 were investigated by TGA. When residual weight (\%) was $90 \%$, the decomposition temperature of Span 60 -IAH was about $28{ }^{\circ} \mathrm{C}$ higher than that of Span60. The wettability is expressed by the water contact angle.

Keywords: Dripping agent, Itaconic anhydride, Span60.

\section{INTRODUCTION}

Surfactant as a kind of functional monomer is being applied in polymer composition. In recent years, types of surfactants are widely used in the chemical industry, food processing, biology, medicine and polyethylene agriculture film ${ }^{1-5}$. The low cost, waste less, easily obtain and other versatile properties were focused by researchers. However, some difficulties appear during application, i.e., some nonionic surfactants can not be expressed dripping ability sufficiently, because of surfactant molecules were flushed by water drop when they are moving on film surface. It will decrease dripping duration, grow cycles of crops can not be satisfied. By analysis this problem, researchers found some reasons, the key point is different types of nonionic surfactants were mixed in linear low density polyethylene (LLDPE) materials in simple way. The simple measure was applied in agriculture film cause serious consequences. However, the chemical structures play a key role in dripping agent and change it to decrease Gibbs free energy of surfactant. If LLDPE was chosen as material for film, the energy of surface should be taking considerate. In order to solve this problem, some investigation has already been carried out ${ }^{6-9}$.

A reactive type nonionic surfactant containing a double bond was synthesized in our laboratory. The chemical structure of reactive type dripping agent was modified by adopted functional monomer. This new structure dripping agent extends the usage and improves properties of polymer film materials. The function of anti-fog is significant either, therefore, it has already become a part of agriculture film production and light transmission has no bad effect. By using this film to cover greenhouse, the whole process of grow cycles without fog or water drop. Reactive dripping agent containing adouble bond can react with active free radical of LLDPE molecular chains, dripping duration of graft film will be maintained for a long time.

In this study, a kind of reactive type dripping agent containing adouble bond was synthesized by using Span60 and itaconic anhydride as main materials. The structure and properties of Span60-IAH were studied systematically.

\begin{tabular}{l}
\hline EXPERIMENTAL \\
\hline Sorbitan monostearate (Span60), $p$-toluenesulfonic acid \\
and hydroquinone were purchased from Tianjin Guangfu Fine \\
Chemical Institute (China). Itaconic anhydride (IAH) was \\
obtained from Shandong Zhongshun Science and Technology \\
Development Co., Ltd (China). Linear low density polyethylene \\
(LLDPE) was produced by Jilin Petrochemical Co., China with \\
the melt flow rate 2 g/10 min. Other chemicals are reagent \\
grade. \\
Preparation of span60-IAH: Span60 $(0.1 \mathrm{~mol})$, itaconic \\
anhydride (0.1 mol), hydroquinone $(0.0097$ mol $), p$-toluene-
\end{tabular}
anhydride $(0.1 \mathrm{~mol})$, hydroquinone $(0.0097 \mathrm{~mol}), p$-toluene- 
sulfonic $(0.0008 \mathrm{~mol})$ were putted in a $250 \mathrm{~mL}$ three necked round bottom flask, equipped with mechanical stirrer and condenser. The temperature was set at $95^{\circ} \mathrm{C}$ for $6 \mathrm{~h}$. The chemical structure of Span60-IAH is shown in Fig. 1.<smiles>C=CC(=O)OC1COC(COC(C)=O)C(O)C1O</smiles>

Fig. 1. Chemical structure of Span60-IAH

Measurement of conversion: Span60-IAH from different reaction time were dissolved in mixture of saturated $\mathrm{NaCl}$ solution (25 wt. \%) and $\mathrm{CCl}_{4}\left(75\right.$ wt. \%) at $95^{\circ} \mathrm{C}$ under vigorous stirring and a nitrogen atmosphere for $8 \mathrm{~h}$, respectively. Keep mixture on the laboratory table for 3 days to make sure solution separate to two layers clearly. Take $1 \mathrm{~mL} \mathrm{NaCl}$ solution layer by using syringe which is $1 \mathrm{~mL}$ volume. Drip 1-2 dropping phenolphthalein test agent in $50 \mathrm{~mL}$ Erlenmeyer flask, then, $0.0041 \mathrm{~mol} / \mathrm{L} \mathrm{KOH}$ solution was dropped in it until pink appear in solution. The consume volume of $\mathrm{KOH}$ solution was recorded. The values of conversion obtained were averages of at least three determinations. The conversion of Span60-IAH was calculated as follows:

$$
\text { Conversion }(\%)=\frac{2 \mathrm{~m}-\mathrm{VCM}}{2 \mathrm{~m}} \times 100 \%
$$

where $\mathrm{m}$ denotes mass of itaconic anhydride ( $\mathrm{mg}), \mathrm{V}$ denotes the consume volume of $\mathrm{KOH}$ solution $(\mathrm{mL}), \mathrm{C}$ denotes the concentrate of $\mathrm{KOH}$ solution ( $\mathrm{mol} / \mathrm{L}), \mathrm{M}$ denotes molar mass of itaconic anhydride $(\mathrm{g} / \mathrm{mol})$.

FT-IR analysis: A Bruker Vertex 70 Fourier-transformed infrared (FT-IR) spectrometer was used for the measurements. The resolution was $4 \mathrm{~cm}^{-1}$ and the number of scans was 16 . FT-IR spectra were used to determine the formation of Span60IAH.

${ }^{1}$ H NMR analysis: ${ }^{1} \mathrm{H}$ NMR spectra were recorded on a Bruker AV $400 \mathrm{MHz}$ Nuclear magnetic resonance (NMR) spectrometer. A sample $(0.08 \mathrm{~g})$ was dissolved in $1.2 \mathrm{~mL}$ of deuterated chloroform $\left(\mathrm{CDCl}_{3}\right)$ in a $\varphi=5 \mathrm{~mm} \mathrm{NMR} \mathrm{tube.}$

Preparation of grafted LLDPE: The grafted LLDPE was prepared in a home-made $\varnothing 30$ twin-screw extruder. LLDPE was pre-irradiated by electron beam accelerator and Span60IAH were premixed, then added into the feeder of extruder. The extrusion temperature was $175^{\circ} \mathrm{C}$. The grafted LLDPE was pelletized.

Measurements of surface tension and contact angles: The surface tension of the Span60-IAH was measured by the drop weight method at $20^{\circ} \mathrm{C}^{10}$. Contact angles were determined on films that were compression-molded from grafted LLDPE. By means of microscrew-driven syringe, $5-8 \mu \mathrm{L}$ of distilled water was added on the film surface. Contact angle measurement was made with a JY-82 contact angle goniometer (Chengde
Test Machine Co., Ltd. China) at ambient humidity and temperature.

TGA analysis: Thermogravimetric analysis (TGA) was investigated with a Perkin-Elmer Pyris 1TGA system under nitrogen atmosphere at a heating rate of $10{ }^{\circ} \mathrm{C} / \mathrm{min}$ from 30 to $400{ }^{\circ} \mathrm{C}$.

\section{RESULTS AND DISCUSSION}

FT-IR spectra of Span60-IAH and Span60 were shown in Fig. 2. Comparing with the spectrum of Span60, the spectrum Span60-IAH showed two new peaks at 1631 and $1722 \mathrm{~cm}^{-1}$. It was clearly seen that one new absorption band at $1722 \mathrm{~cm}^{-1}$, which was attributed to contribution of carboxyl groups of itaconic anhydride esterification reaction after and a peak at $1631 \mathrm{~cm}^{-1}$ which was attributed to contribution of double bond of itaconic anhydride. These results indicated that itaconic anhydride has been successfully reacted onto the Span60 molecular.

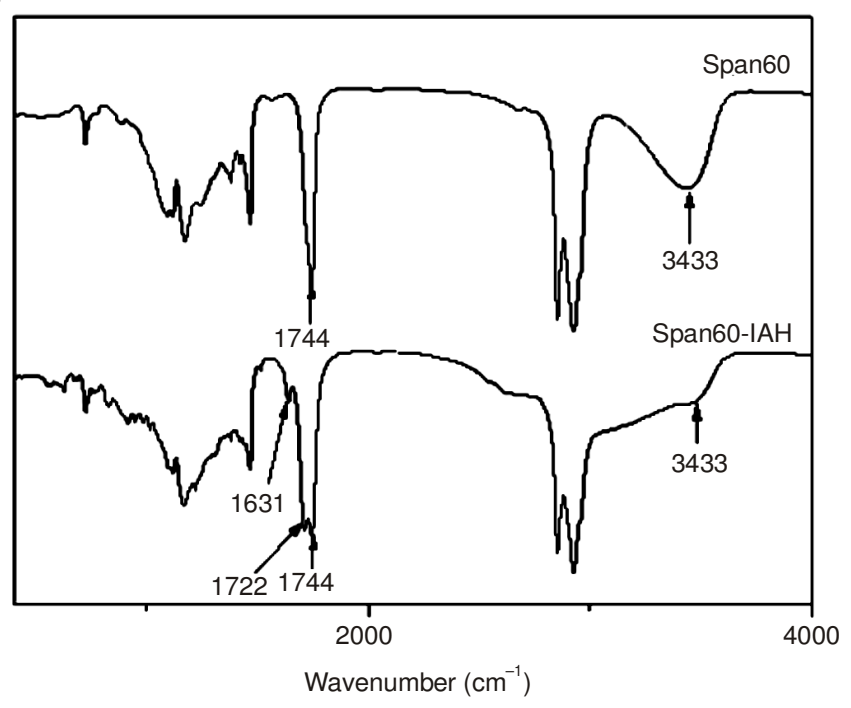

Fig. 2. FTIR spectra of Span60 and Span60-IAH

${ }^{1}$ H NMR spectra of Span60-IAH and Span60 were shown in Fig. 3. ${ }^{1} \mathrm{H}$ NMR spectrum of Span60-IAH showed chemical shift peaks at $5.23 \mathrm{ppm}$, which are attributed to chemical shift of ${ }^{1} \mathrm{H}$ in $\mathrm{H}_{2} \mathrm{C}=\mathrm{C}$ and chemical shift peaks at $3.36 \mathrm{ppm}$ and $3.61 \mathrm{ppm}$, which are attributed to chemical shift of ${ }^{1} \mathrm{H}$ in $\mathrm{C}=\mathrm{CCH}_{2}$. This indicated that itaconic anhydride has been reacted onto Span60 molecular.

The effect of reaction time on conversion was shown in Fig. 4. It could be seen that the conversion increase with increasing reaction time. When the time was 5 and $6 \mathrm{~h}$, the conversion of Span60-IAH were 99.1 and $99.4 \%$, respectively. This result suggested that itaconic anhydride reaction with Span60 was basically complete after reaction time $5 \mathrm{~h}$.

When the concentration of the surface active material was close to the critical micelle concentration $\left(\mathrm{C}_{\mathrm{CMC}}\right)$, the adsorption changed to saturation $\left(\Gamma_{\mathrm{M}}\right)^{11}$. The relationship between surface tension $(\gamma)$ and the concentration $(\mathrm{C})$ could be expressed as:

$$
\mathrm{d} \gamma=-2.3 \mathrm{~K} \Gamma_{\mathrm{M}} \mathrm{RT} d \operatorname{lgC}
$$

The surface tension $(\gamma)$ and the logarithm of the concentration could be obtained ( $-\mathrm{d} \gamma / d \operatorname{lgC}=\mathrm{k}$, a constant $)$. As a rule, 


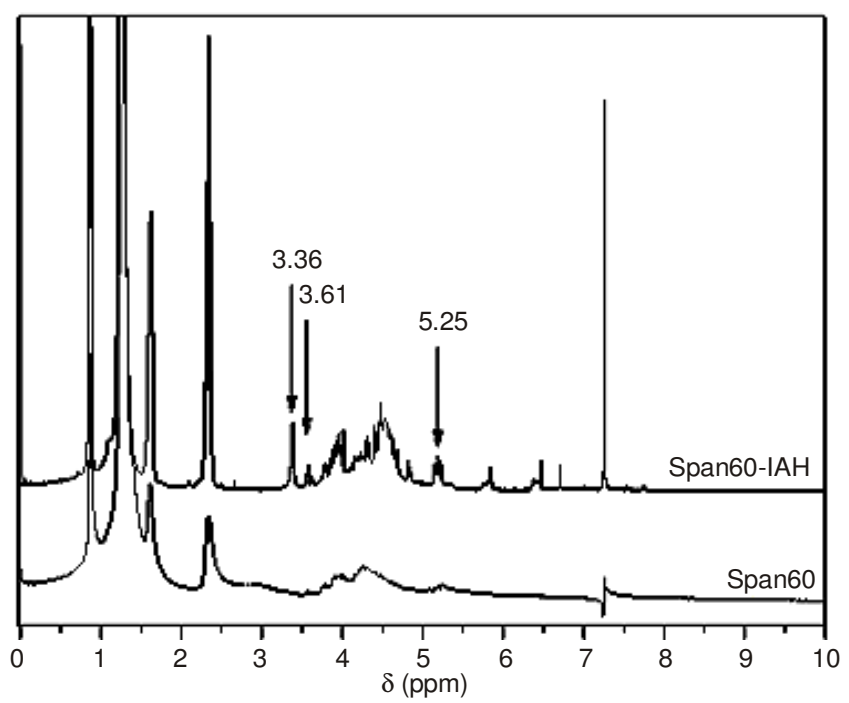

Fig. 3. ${ }^{1} \mathrm{H}$ NMR spectra of Span60-IAH and Span60

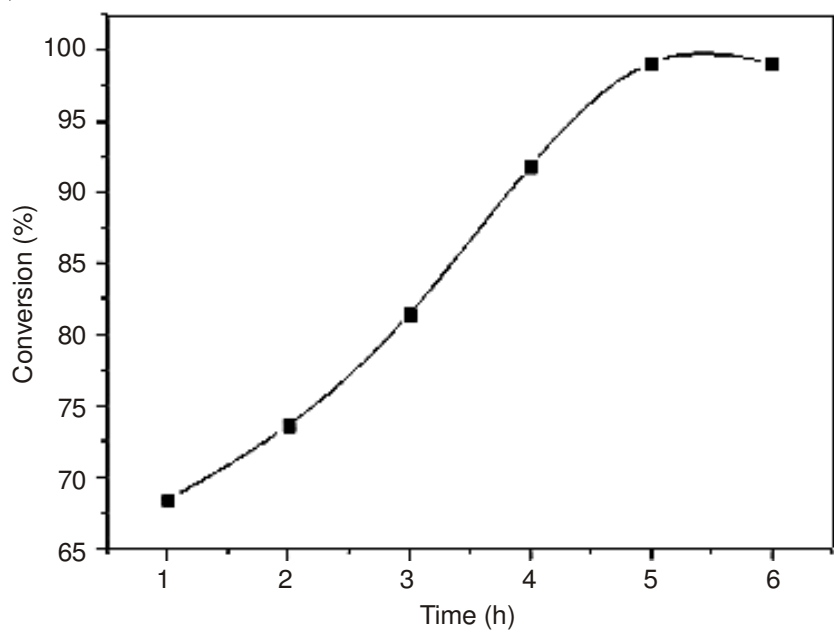

Fig. 4. Eeffect of time on the conversation of Span60-IAH

the surface adsorption would be close to saturation as the surface tension decreased to $20 \mathrm{~N} \mathrm{~m}^{-1}$. Furthermore, if surface tension $\gamma$ and concentration $\mathrm{C}$ were introduced into eqn. 1, the following equation would be obtained:

$$
\begin{gathered}
-\Delta \gamma=\left(\gamma_{0}-\gamma\right)+\left(\gamma-\gamma_{\text {СMC }}\right) \\
-\Delta \gamma=20+2.3 K \Gamma_{\mathrm{M}} \mathrm{RT} \operatorname{Tg}\left(\mathrm{C}_{\mathrm{CMC}} / \mathrm{C}_{20}\right)
\end{gathered}
$$

where $-\Delta \gamma$ denoted the whole reduction of the surface tension, when the $\mathrm{C}_{\mathrm{CMC}}$ was reached. $\Gamma_{\mathrm{M}}$ was the maximum adsorption and $\mathrm{K}$ was a constant. The $\gamma_{0}$ and $\gamma_{\mathrm{CMC}}$ were the surface tension of the solvent and solution, respectively.

The compound Span60-IAH is a product of Span60 reacted with itaconic anhydride. During measurement surface tension, the solubility of Span60-IAH and Span60 in water showed no obvious difference, but the surface tension of Span60-IAH decreased markedly. The surface activity data of Span60-IAH in water was shown in Table-1. This suggests that the activity of the surfactant containingadouble bond and carboxyl groups was stronger than that of Span60. The result was in accordance with the aforementioned theory.

The thermal stability of Span60-IAH and Span60 were investigated by TGA and the results are depicted in Fig. 5. It could be seen that the thermal stability of Span60-IAH and Span60 were similar, before $175^{\circ} \mathrm{C}$. However, when residual
TABLE-1

PARAMETERS $\gamma_{\mathrm{CMC}}, \mathrm{C}_{\mathrm{CMC}}, \Delta \gamma$ of SPAN60 AND SPAN60-IAH

\begin{tabular}{lccc}
\hline \multicolumn{1}{c}{ Samples } & $\begin{array}{c}\gamma_{\text {CMC }} \\
\left(10^{3} \mathrm{~N} \mathrm{~m}^{-1}\right)\end{array}$ & $\begin{array}{c}\mathrm{C}_{\mathrm{CMC}} \\
\left(10^{-5} \mathrm{~mol} \mathrm{~L}^{-1}\right)\end{array}$ & $\begin{array}{c}\Delta \gamma \\
\left(10^{3} \mathrm{~N} \mathrm{~m}^{-1}\right)\end{array}$ \\
\hline Span60 & 49.02 & 1.62 & - \\
Span60-IAH & 27.49 & 0.91 & 21.51 \\
\hline$\Delta \gamma=\gamma_{\text {CMC }}-\gamma$ Span60 & & &
\end{tabular}

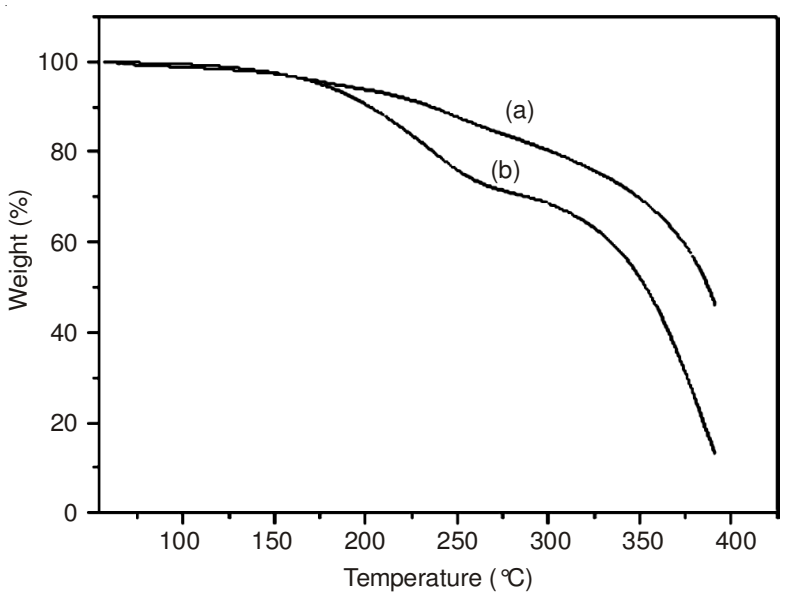

Fig. 5. TGA curves of Span60-IAH (a) and Span60 (b)

weight (\%) was $95 \%$, the decomposition temperature of Span60-IAH was about $18{ }^{\circ} \mathrm{C}$ higher than that of Span60. When residual weight (\%) was $90 \%$, the decomposition temperature of Span60-IAH was about $28{ }^{\circ} \mathrm{C}$ higher than that of Span60, which demonstrated that Span60-IAH exhibited a better thermal stability than that of Span60.

Contact angles on film surfaces of LLDPE- $g$-(Span60IAH) with different monomer concentration with water as the medium were shown in Table-2. When monomer concentration was 0.5 and 2 wt. $\%$, the contact angles of water were $63^{\circ}$ and $29^{\circ}$, respectively. Observe that with an increasing concentration of Span60-IAH, the contact angles of water decrease. This result suggests that the surface polarity of films is augmented by an increase of monomer concentration in grafted LLDPE.

\begin{tabular}{ccc}
\multicolumn{3}{c}{ TABLE-2 } \\
CONTACT ANGLES OF WATER DROP ON \\
FILM SURFACES OF LLDPE- $g$-(SPAN60-IAH) \\
\hline Samples & $\begin{array}{c}\text { Monomer } \\
\text { concentration }(\text { wt. } \%)\end{array}$ & $\begin{array}{c}\text { Contact } \\
\text { angle }\left(^{\circ} \text { ) }\right.\end{array}$ \\
\hline LLDPE- $g$-(Span60-IAH) & 0.5 & 63 \\
LLDPE- $g$-(Span60-IAH) & 1.0 & 46 \\
LLDPE- $g$-(Span60-IAH) & 1.5 & 34 \\
LLDPE- $g$-(Span60-IAH) & 2.0 & 29 \\
\hline
\end{tabular}

\section{Conclusion}

A reactive type dripping agent containing adouble bond, Span60-IAH was synthesized. When the time was $6 \mathrm{~h}$, the conversion of Span60-IAH reached $99.4 \%$. The activity of the reactive type dripping agent containingadouble bond and carboxyl groups was stronger than that of Span60. The results of TGA measurement demonstrated that Span60-IAH exhibited a better thermal stability than Span60. The surface polarity of films is augmented by an increase of monomer concentration in grafted LLDPE. 


\section{ACKNOWLEDGEMENTS}

The authors would like to acknowledge the financial support of Support Project of National Science and Technology of China (Project No 2012BAD11B01-3).

\section{REFERENCES}

1. R. Mahfud, T. Agag, H. Ishida, S. Shaikh and S. Qutubuddin, J. Colloid Interf. Sci., 407, 339 (2013).

2. Y. Yang and D.J. Mcclements, Food Hydrocoll., 30, 712 (2013).

3. H.K. Ma, M.M. Liu, S.Y. Li, Q. Wu, J.C. Chen and G.Q. Chen, J. Biotechnol., 166, 34 (2013).

4. G.K. German, E. Pashkovski and E.R. Dufresne, J. Biomech., 46, 2145 (2013).
5. W.F. Li, Z.H. Yao, R.X. Yao and S.M. Liu, Polym-Plastic. Tech. Eng., 52, 603 (2013)

6. L. Zhang, X. Lv, Y.J. Zhu, J. Zhang, H. Wang and Y.B. Tan, Colloid Polym. Sci., 289, 1579 (2011).

7. N.A. Negm, A.F.M. El Farargy, D.E. Mohammed and H.N. Mohamad, J. Surfact. Deterg., 15, 433 (2012).

8. H.J. Xu, D.D. Chen and Z.G. Cui, J. Surfact. Deterg., 14, 167 (2011).

9. N.A. Negm, N.G. Kandile and M.A. Mohamad, J. Surfact. Deterg., 14, 325 (2011).

10. S. Wu, Polymer Interface and Adhesion, Marcel Dekker, Inc.: New York, p. 15 (1982).

11. M.J. Rosen, Surfactants and Interfacial Phenomena, Wiley Interscience, New York, p. 295 (1978). 